\title{
Femtosecond Spectral Hole Burning Spectroscopy as a Probe of Exciton Dynamics in Quantum Dots
}

\author{
T. Müller*, T. Moldaschl, W. Parz, S. Golka, \\ G. Strasser and K. Unterrainer \\ Institute of Photonics and Center for Micro- and Nanostructures \\ Vienna University of Technology, A-1040 Vienna, Austria
}

\begin{abstract}
We present measurements of the dynamics of photoexcited excitons in an ensemble of InAs/GaAs self-assembled quantum dots using a femtosecond spectral hole burning technique. We use this technique to examine the exciton spin relaxation and the line shape of optical transitions in the dots.
\end{abstract}

PACS numbers: 73.21.La, 78.47.-p, 78.67.Hc

\section{Introduction}

The atom-like character of excitonic states in semiconductor quantum dots (QDs) allows translating many concepts known from atomic physics to the solid state [1]. However, in contrast to atoms, QDs exhibit a strong coupling to their environment (especially at elevated temperatures), leading to population and phase relaxation on picosecond - or even sub-picosecond - timescales. A detailed understanding of these processes is essential for the design of future quantum information processing devices, as well as novel optoelectronics.

The strong inhomogeneous broadening of interband transitions in QDs makes it difficult to selectively excite/probe excitonic transitions in the dots without exciting/probing other exciton complexes, such as biexcitons or charged excitons. Time-resolved studies of individual excitons in single quantum dots are possible, however, such measurements are challenging and in many cases suffer from a low signal-to-noise ratio and a bad time resolution. Therefore, an alternative technique - ultrafast spectral hole burning (SHB) spectroscopy - is presented, which provides good energy resolution, sub-picosecond time resolution, and high signal-to-noise ratios.

*corresponding author; e-mail: thomas.mueller@tuwien.ac.at 


\section{Femtosecond spectral hole burning spectroscopy}

The QD sample used in this study consists of 30 layers of InAs self-assembled QDs embedded in a GaAs matrix. The dot density was estimated from atomic force microscopy measurements to be approximately $2 \times 10^{10} \mathrm{~cm}^{-2}$ per layer. In order to be accessible to our Ti:sapphire laser system, the exciton transitions were shifted to higher energies by rapid thermal annealing $\left(240 \mathrm{~s}, 800^{\circ} \mathrm{C}\right)$. In addition, both sides of the sample were coated with antireflection coatings composed of $\lambda / 4$-SiN layers. Low-temperature $(T=5 \mathrm{~K})$ photoluminescence from the excitonic ground state of the dot ensemble is observed at $1281 \mathrm{meV}$. The inhomogeneous broadening of the transitions is $\approx 45 \mathrm{meV}$ (full width at half maximum, FWHM) and reflects the size distribution of the dots. The energy level diagram of the excitonic $s$-shell is shown in the inset of Fig. 1. The (slightly non-degenerate) exciton states $X$ and $Y$ can be excited by linearly polarized light along the (110) and $(-110)$ directions, respectively. $X X$ denotes the biexciton state, whose energy is less than twice the bare exciton energy, the difference between them being the biexciton binding energy $\Delta E$.

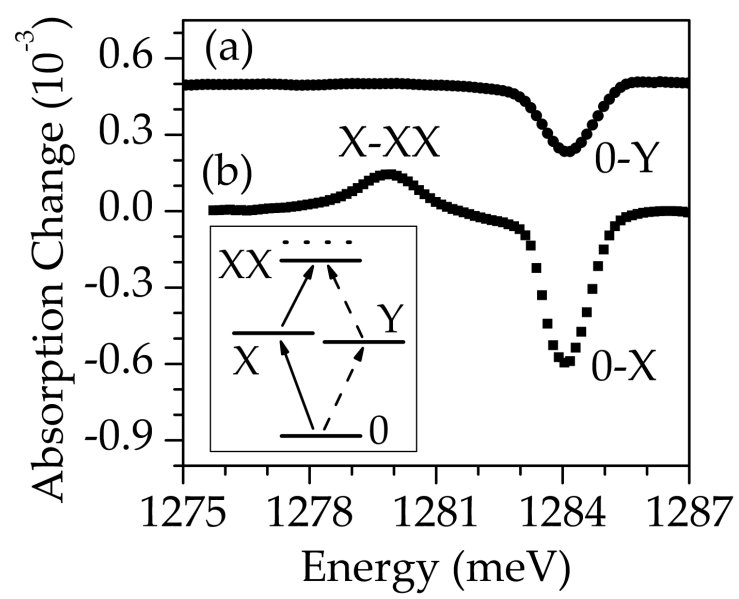

Fig. 1. SHB signals for $(a)$ perpendicular and $(b)$ parallel polarizations of pump and probe pulses. The polarization of the pump was set in both measurements parallel to the (110) direction of the sample. Inset: energy level diagram of a QD. The polarization selection rules for optical transitions are indicated by solid and dashed lines.

SHB measurements were performed using a mode-locked Ti:sapphire laser that delivers $80 \mathrm{fs}$ pulses with a center frequency of $1285 \mathrm{meV}$ and a spectral width of $\approx 14 \mathrm{meV}$ (FWHM). A grating pulse shaper, consisting of a grating pair, a lens pair and a slit assembly, was used to generate the narrow band-pump pulse. An adjustable attenuator allowed continuous variation of the excitation intensity. The pump induced absorption change was measured with a weak ( $4 \%$ of the laser 
intensity) broad band-probe. The probe was spectrally dispersed with a 3/4-meter grating monochromator which allowed the determination of absorption changes induced at photon energies different from that of the pump. The temporal delay between the pump and probe pulses was controlled by a motorized translation stage. In addition, half-wave-plates were used to independently adjust the (linear) polarizations of pump and probe pulses.

Figure 1 shows SHB signals recorded at $T=5 \mathrm{~K}$. In these measurements a pump pulse polarized parallel to the (110) direction of the sample was tuned to the maximum of the excitonic ground state luminescence. The linear polarization of the broad band-probe was set either perpendicular $(a)$ or parallel $(b)$ to that of the pump, and the differential absorption change of the probe was measured at a temporal delay of approximately 10 ps after excitation. The excitation band width of the pump pulse was set to $1.4 \mathrm{meV}$ (FWHM). Both measurements show reduced absorption at the pump photon energy, corresponding to a bleaching of the $0-X$ and $0-Y$ transitions. In the co-polarized case $(b)$, induced absorption (a socalled antihole) is observed which is due to the transition from the exciton state $X$ to the biexciton state $X X$ at an energy of $\Delta E \approx 4 \mathrm{meV}$ below the exciton peak [2].

\section{Exciton spin relaxation}

The temporal evolution of the antihole at $T=90 \mathrm{~K}$ is shown in Fig. 2a. With increasing pump-probe delay, the ratio of cross-polarized signal to co-polarized signal increases and reaches one for long delay times. The development of an antihole in the cross-polarized case is due to spin relaxation: As $X$ excitons flip their spin, the $Y$ exciton state gets populated, giving rise to $Y-X X$ absorption [3]. Since the splitting between the two exciton states $E_{X}-E_{Y}$ is much smaller than $k_{\mathrm{B}} T$ (even at $T=5 \mathrm{~K}$ ), relaxation from $X$ to $Y$ is comparable to relaxation from $Y$ to $X$. Further, it has been shown that the optical transition strengths and lifetimes for the two excitonic transitions are similar [4]. Under those assumptions a simple rate equation model [5] yields $\left(A_{\|}-A_{\perp}\right) /\left(A_{\|}+A_{\perp}\right) \propto \exp \left(-2 \Gamma_{\text {spin }} \tau\right)$, from which the spin relaxation rate $\Gamma_{\text {spin }}$ can be deduced. Here, $A_{\|}$and $A_{\perp}$ denote the antihole amplitudes for co- and cross-polarized probe light, respectively, and $\tau$ is the pump-probe delay time. From the difference of the co- and cross-polarized antihole magnitudes, we obtain $1 / \Gamma_{\text {spin }}=90 \mathrm{ps}$. For comparison, the measured lifetimes of the exciton states are $\approx 0.5$ ns. Let us note that a single carrier (electron or hole) spin flip would turn the QD into a dark state to which our experiment is not sensitive. Thus, $\Gamma_{\text {spin }}$ describes an exciton spin flip process, where both electron and hole simultaneously flip their spin. The spectral hole in our experiment exhibits the same behavior as the antihole.

In order to gain information on the origin of the exciton spin relaxation we performed temperature dependent measurements. Figure $2 \mathrm{~b}$ presents the temperature dependence of the relaxation time. It increases rapidly from $90 \mathrm{ps}$ to 


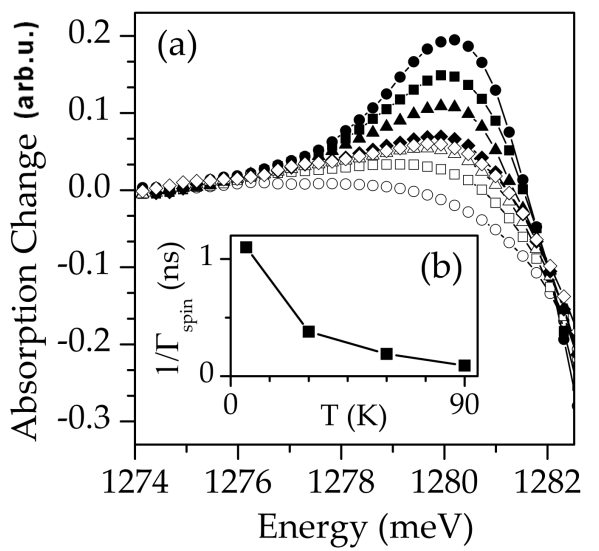

Fig. 2. (a) Temporal evolution of the spectral antihole for perpendicular (open symbols) and parallel (filled symbols) polarizations of pump and probe pulses at $T=90 \mathrm{~K} . \tau=$ $10 \mathrm{ps}$ (circles), $30 \mathrm{ps}$ (squares), $50 \mathrm{ps}$ (triangles), and $90 \mathrm{ps}$ (diamonds). (b) Temperature dependence of the exciton spin relaxation time.

$1.15 \mathrm{~ns}$ as the temperature is reduced from $90 \mathrm{~K}$ to $5 \mathrm{~K}$. At low temperature, the spin relaxation time is more than twice longer than the exciton lifetime which remains approximately constant over the investigated temperature range. The large change of the spin relaxation time suggests that phonon scattering related mechanisms become significant at high temperature. The observed temperature dependence is characterized by a small activation energy which strongly points to an acoustic phonon mediated spin flip process. Fundamental theoretical studies on the exciton spin flip in QDs are rare. Tsitsishvili presented calculations, where sequential acoustic phonon assisted flips of the electron and hole spins, mediated by spin-orbit coupling, results in a transition between the bright exciton states [6]. They obtained relaxation times on the order of $\approx 1$ ns (i.e. comparable to our experimental results) at low temperature, and their calculated temperature dependence closely resembles that of our measurement.

\section{Acoustic phonon side bands of excitonic transitions}

In order to gain more insight in the line shape of the optical transitions, we recorded SHB spectra by setting the band width of the pump pulse to only $350 \mu \mathrm{eV}$ (FWHM). Spectra were recorded at different temperatures and are shown in Fig. 3.

At $T=5 \mathrm{~K}$, the SHB line shape resembles that of the pump pulse. However, with increasing temperature, a broad background is observed on both sides of the pump. This feature is observed best at $T=60 \mathrm{~K}$. In accordance with single-QD photoluminescence experiments $[7,8]$, we assign the central line to the excitonic zero-phonon transition (broadened by the spectral width of the pump), and the broad background to coupled exciton-acoustic phonon side bands. The spectral 


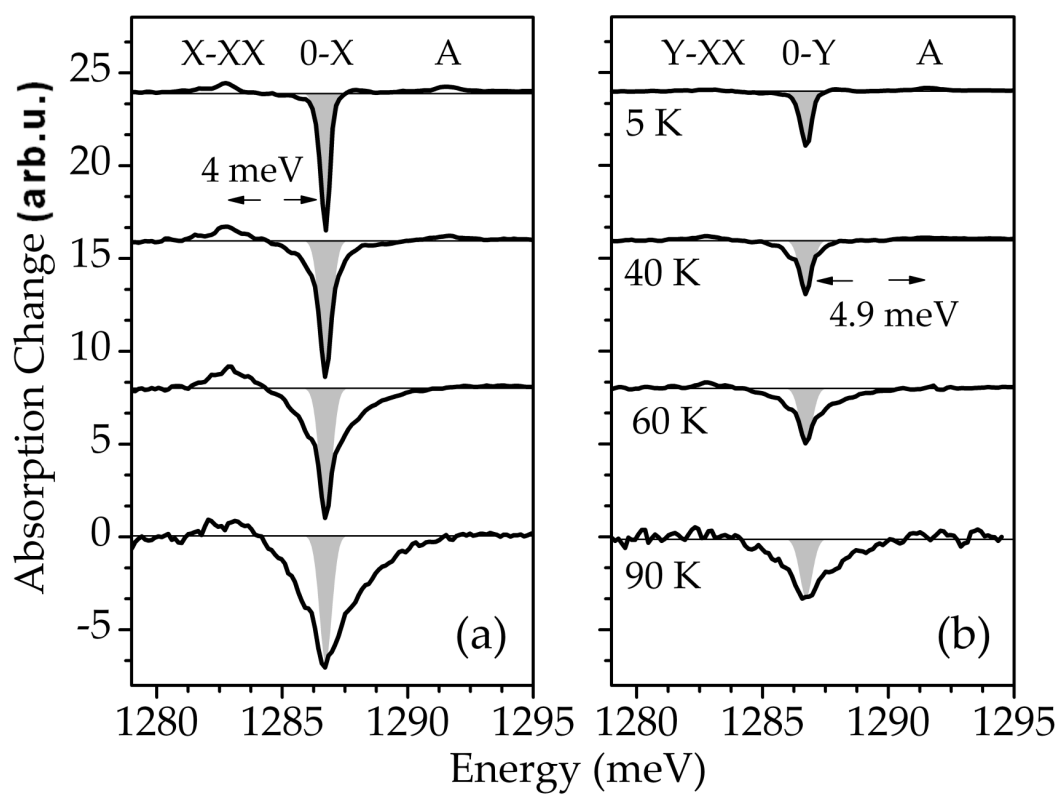

Fig. 3. SHB signals for (a) parallel and (b) perpendicular polarizations of pump and probe pulses. The shape of the pump is symbolized by the gray areas. At elevated temperatures the shape of the spectral hole strongly deviates from that of the pump pulse.

width of the phonon side bands is approximately independent of temperature. It is determined by the wave vector $q$ of acoustic phonons which can interact with the QDs: it is limited to about twice the inversion localization length $\xi$, with the strongest coupling for phonons with wave vector $q \sim 1 / \xi[7]$. With rising temperature the ratio between the area of the phonon side band and that of the zero-phonon line increases, and at $T=90 \mathrm{~K}$ the zero-phonon line cannot be separated from the side bands anymore. Since the energy level separation in our QDs is larger than typical phonon energies, inelastic scattering events are strongly suppressed. Therefore, the loss of phase coherence is mainly due to elastic interaction with acoustic phonons - a process which is not accompanied by energy relaxation - leading to pure dephasing. Another feature (labelled $A$ ) is observed $\approx 4.9 \mathrm{meV}$ above the pump photon energy. Its origin is not clarified yet.

\section{Acknowledgments}

This work was supported by the Austrian Fonds zur Förderung der Wissenschaftlichen Forschung (SFB-ADLIS, SFB-IRON) and the ECNoE program SANDiE. 


\section{References}

[1] See, for example, Single Quantum Dots, Ed. P. Michler, Springer, Berlin 2003 and references therein.

[2] A.S. Lenihan, M.V. Gurudev Dutt, D.G. Steel, S. Ghosh, P. Bhattacharya, Phys. Rev. B 69, 045306 (2004)

[3] T. Müller, G. Strasser, K. Unterrainer, Appl. Phys. Lett. 88, 192105 (2006).

[4] J.R. Guest, T.H. Stievater, Xiaogin Li, Jun Cheng, D.G. Steel, D. Gammon, D.S. Katzer, D. Park, C. Ell, A. Thränhardt, G. Khitrova, H.M. Gibbs, Phys. Rev. B 65, 241310 (2002).

[5] A. Tackeuchi, R. Ohtsubo, K. Yamaguchi, M. Murayama, T. Kitamura, T. Kuroda, T. Takagahara, Appl. Phys. Lett. 84, 3576 (2004).

[6] E. Tsitsishvili, R. v. Baltz, H. Kalt, Phys. Rev. B 72, 155333 (2005).

[7] L. Besombes, K. Kheng, L. Marsal, H. Mariette, Phys. Rev. B 63, 155307 (2001).

[8] E. Peter, J. Hours, P. Senellart, A. Vasanelli, A. Cavanna, J. Bloch, J.M. Gérard, Phys. Rev. B 69, 041307(R) (2004). 